\title{
Molecular Mechanisms of Therapies for Prostate Cancer with Bone Metastasis
}

\author{
Alexandra Vander Ark, Erica Woodford and Xiaohong $\mathrm{Li}^{*}$ \\ Program for Skeletal Disease and Tumor Microenvironment, Center for Cancer and Cell Biology, Van Andel Institute, Grand Rapids, MI, \\ 49503, USA
}

\begin{abstract}
Prostate cancer $(\mathrm{PCa})$ is one of the most diagnosed cancers in American men and is a leading cause of cancer deaths in advanced forms of disease. Seventy to ninety percent of men who die of PCa will have developed bone metastases during the course of the disease, and these bone metastases cause severe pain that can lead to further skeletal complications. The standard treatment for PCa generally includes androgen deprivation therapy, radiation, and chemotherapy, either singly or in combination, depending on disease progression. These treatments are able to slow disease progression slightly, but an urgent need remains for a more curative approach. In addition to the adverse effects resulting from these treatments, androgen deprivation therapy can often lead to a castration-resistant form of the disease, which is even more difficult to treat and often becomes metastatic. In the past several years, new drugs that target androgens, the bone microenvironment, and other mechanisms involved in $\mathrm{PCa}$ have shown promise in clinical trials for advanced $\mathrm{PCa}$; these include abiraterone, enzalutamide, sipuleucel-T, radium-223, denosumab, and cabazitaxel, and will be described in further detail in this review. The continuous improvement upon current therapies and development of new drugs is promising for the future of advanced PCa, and the authors will give their perspective on these different treatment approaches.
\end{abstract}

\section{Introduction}

According to the American Cancer Society, 1 in 7 men will be diagnosed with prostate cancer (PCa) in their lifetime. ${ }^{1}$ There is a 10 -year survival rate of $99 \%$ when the disease is caught early; however, this rate drops to $30 \%$ when patients develop metastasis. Seventy to ninety percent of patients who die of PCa have

Keywords: Prostate cancer; Bone metastasis; Treatment; Androgen deprivation therapy; Radiation; Bone microenvironment; Androgen; Castration-resistant.

Abbreviations: PCa, prostate cancer; ADT, androgen deprivation therapy; AR, androgen receptor; CRPC, castration-resistant prostate cancer; LHRH, luteinizing hormone-releasing hormone; PSA, prostate-specific antigen; RANKL, receptor activato of nuclear factor kappa-B ligand; U.S. FDA, United States Food and Drug Administration; SREs, skeletal-related events.

Received: 30 August 2016; Revised: 29 September 2016; Accepted: 08 October 2016 DOI: 10.14218/JERP.2016.00023

*Correspondence to: Xiaohong Li, Program for Skeletal Disease and Tumor Microenvironment, Center for Cancer and Cell Biology, Van Andel Research Institute, 333 Bostwick Ave., N.E., Grand Rapids, MI 49503, USA. Tel: 1-616-234-5267, E-mail: xiaohong.li@vai.org bone metastasis, which classifies their disease state as advanced. ${ }^{2}$ PCa bone metastasis causes skeletal-related events (SREs), such as fracture, hypercalcemia, or spinal cord compression, as well as severe pain. Current therapies, such as bisphosphonates, steroids, and chemotherapies, are used to relieve these symptoms and slow disease progression, but they are also associated with adverse reactions and do not significantly improve the patients' overall survival. Therefore, developing new approaches to target PCa bone metastasis is an urgent need.

It is important to understand the molecular mechanisms behind $\mathrm{PCa}$ metastasis when developing new therapies. Normal bone homeostasis is largely dependent on osteoclast and osteoblast function during bone turnover, as well as the various factors that are released during the process. PCa cells secrete factors such as parathyroid hormone-related protein (PTHrP), which initiates osteoblast expression of the receptor activator of nuclear factor kappa-B ligand (RANKL), which further stimulates maturation of osteoclasts to resorb bone. The bone is a powerhouse, which stores many growth factors and cytokines that are released upon PCainduced resorption. Factors like transforming growth factor-beta (TGF- $\beta$ ) are released from the bone matrix and cause stimulation of osteoblasts, which, in turn, signal to osteoclasts for more bone resorption. This rapid bone turnover that occurs during PCa bone metastasis is known as the "vicious cycle".

This review will discuss the molecular mechanisms of current clinical therapies and our perspectives on the potential new therapies for PCa bone metastasis patients. There have been several drugs approved for PCa therapy in the last few years, targeting a variety of cellular pathways. We will focus on the main approaches to current $\mathrm{PCa}$ therapy, primarily on the respective underlying molecular mechanisms of each treatment. A list of drugs discussed in this review along with their respective categories and mechanisms of action are included in Table 1 .

\section{Targeting cancer cells}

\section{Androgen ablation therapy}

The surgical or chemical removal of androgen signaling, known as androgen deprivation therapy (ADT), is a common treatment for advanced $\mathrm{PCa}$. ADT reduces the amount of available androgen that would otherwise stimulate PCa cell growth through the androgen receptor (AR). Administration of ADT prior to primary tumor removal is still controversial, but studies have shown that it leads to a decrease in lymph node invasion and better organ confinement as well as overall progression-free survival when initiated at diagnosis. ${ }^{3}$ Some clinicians choose a combination treatment of pelvic 
Vander Ark A. et al: Therapeutics in PCa Bone Metastasis

Table 1. Mechanistic actions of prostate cancer therapeutics

\begin{tabular}{|c|c|c|}
\hline Drug Name & Therapeutic Category & Mechanism of Action \\
\hline Abiraterone & Androgen Deprivation Therapy & CYP17 inhibitor that decreases circulating levels of androgen \\
\hline Cabitaxel & Chemotherapeutic & $\begin{array}{l}\text { Antimitotic that stops PCa division and alters the cell efflux pump to extend } \\
\text { cellular drug levels }\end{array}$ \\
\hline CAR T Therapy & Immune Therapy & Targets prostate-specific antigens such as PSMA or PSCA to kill PCa cells \\
\hline Degarelix & LHRH Antagonist & $\begin{array}{l}\text { Stops LHRH from binding its receptor and prevents LH flare that would otherwise } \\
\text { spike testosterone levels }\end{array}$ \\
\hline Denosumab & RANKL Inhibitor & RANKL antibody that blocks osteoclast differentiation and activity \\
\hline Docetaxel & Chemotherapeutic & Antimitotic agent that stops $\mathrm{PCa}$ cell division \\
\hline Enzalutamide & Androgen Deprivation Therapy & $\begin{array}{l}\text { Androgen receptor inhibitor that prevents AR translocation to the nucleus and } \\
\text { further androgen signaling }\end{array}$ \\
\hline $\begin{array}{l}\text { Galeterone and } \\
\text { Niclosamide }\end{array}$ & Androgen Deprivation Therapy & $\begin{array}{l}\text { Targets AR-V7 to reduce androgen levels (generally used in combination with } \\
\text { either abiraterone or enzalutamide) }\end{array}$ \\
\hline Ipilimumab & Immune Checkpoint Therapy & $\begin{array}{l}\text { CTLA- } 4 \text { antibody that promotes adaptive immunity and T cell response to attack } \\
\text { PCa cells }\end{array}$ \\
\hline $\begin{array}{l}\text { Nivolumab and } \\
\text { Pembrolizumab }\end{array}$ & Immune Checkpoint Therapy & PD-1 antibody that allows immune response of cytotoxic T cells to attack PCa cells \\
\hline Radium-223 & Radiation Therapy & $\begin{array}{l}\text { Alpha-emitting radiation with structure similar to calcium for greater bone } \\
\text { specificity }\end{array}$ \\
\hline Sipuleucel-T & Immunotherapy & $\begin{array}{l}\text { Cancer vaccine that facilitates the T cell immune response targeting PAP on PCa } \\
\text { cells }\end{array}$ \\
\hline SRPK1 Inhibitor & VEGF and FGFR Inhibitors & Alters VEGF splicing to antiangiogenic isoforms, reducing tumor angiogenesis \\
\hline Zoledronic Acid & Bisphosphonate & Targets osteoclasts to reduce bone resorption and turnover \\
\hline
\end{tabular}

radiation and ADT for their patients to target any micrometastases that may be present at the time of primary tumor removal. ${ }^{4}$

One disadvantage to ADT is that a flare reaction in bone metastasis can occur upon initiation, leading to rapid bone repair and an increase in osteoblast activity. ${ }^{5}$ This makes it very important for clinicians to take into account the progression of disease and risk for the patient before beginning treatment. While ADT can slow progression of disease, there are adverse effects for patients, including hot flashes, night sweats, hair loss, irritability, loss of libido, and erectile dysfunction. ${ }^{6}$ Another disadvantage to ADT is that testosterone levels have to be monitored consistently, which becomes difficult when trying to detect castration levels of $50 \mathrm{ng} /$ $\mathrm{dL}$ or less. The testosterone level of $50 \mathrm{ng} / \mathrm{dL}$ has been set as the standard for castration, but there is question as to whether the baseline should be reduced to $20 \mathrm{ng} / \mathrm{dL}$, for which better patient results have been reported. ${ }^{3}$

Drugs like abiraterone, enzalutamide, and galeterone, have been developed to target alternate androgen activation of AR that may contribute to rising testosterone levels and PCa progression. ${ }^{7-9}$ The first of these drugs, abiraterone, is a CYP17 inhibitor that prevents the conversion of pregnenalone to dihydrotestosterone (DHT) and has been shown to significantly increase survival, particularly in patients with metastatic castration-resistant PCa (CRPC), as compared to treatment with prednisone alone. ${ }^{9}$ The second of these drugs, enzalutamide, works through an AR binding competition mechanism and inhibition of translocation of AR into the nucleus, both of which impair AR activation. ${ }^{8}$ Both abiraterone and enzalutamide have improved survival in patients, but one-third of $\mathrm{PCa}$ patients have an AR-V7 splice variant that makes them resistant to this therapy. In order to overcome resistance to these drugs, a couple of new therapies are in trials, including niclosamide and galeterone, and have shown some success by specifically targeting
AR-V7 along with inhibition of AR by abiraterone or enzalutamide. ${ }^{10}$ These alternatives could be used to reduce androgen signaling ever further, but, even so, ADT does not cure prostate cancer and can lead to castration-resistant disease.

Luteinizing hormone-releasing hormone (LHRH) agonists and antagonists are another way to reduce androgen signaling in PCa patients. LHRH agonists act on the anterior pituitary gland to block testosterone production. ${ }^{3}$ The down side of this agonist is that it causes an initial spike in $\mathrm{LH}$ release that can stimulate $\mathrm{PCa}$ cells, leading to side effects like spinal cord compression, ureteral/ urethral obstruction, or bone pain. In this case, antiandrogens are generally added to the therapy to block downstream AR signaling and suppress these side effects. This strategy of combined androgen blockade is used to keep the levels of androgens below the castration baseline of $50 \mathrm{ng} / \mathrm{dL}$, even with the LH burst from drug initiation.

Combined therapies can help keep testosterone levels at bay but come with added risk to patients, including increased risk for diabetes, osteoporosis, cardiac events and stroke. ${ }^{3}$ LHRH antagonists have been more recently investigated for their ability to help avoid LH flare from the pituitary gland, meaning that no antiandrogens would be needed to counteract the increase in testosterone and ultimately equating to less risk for adverse effects. Degarelix is an LHRH antagonist that works by consistently suppressing folliclestimulating hormone (FSH) levels, which would otherwise stimulate PCa growth. LHRH antagonists have been found to improve progression-free survival better than other ADTs as well. ${ }^{3}$

While these therapies can reduce androgen signaling to $\mathrm{PCa}$ cells and slow down progression of disease, patients and clinicians have to decide on the best treatment for their individual case. Since ADT can lead to CRPC and metastasis, a patient with low-risk disease has to acknowledge the potential for future complications 
associated with starting ADT. Once PCa becomes castrationresistant or metastatic, it is very difficult to treat and most often leads to patient death.

\section{Chemotherapy}

Taxanes are a group of chemotherapeutic agents used to treat advanced $\mathrm{PCa}$ that interfere with microtubules during mitosis and prevent the cell from reaching anaphase, thereby leading to apoptosis. ${ }^{11}$ Docetaxel was approved for treatment of metastatic CRPC in 2004 and has since become the standard treatment for advanced disease, but, unfortunately, disease progression is still inevitable. Docetaxel used in combination with other therapies has provided some promising results of progression-free survival. ${ }^{7,11,12}$ Even so, some PCa becomes resistant to docetaxel and new treatment options are needed. Cabazitaxel was approved by the U.S. Food and Drug Administration (FDA) to treat docetaxel-resistant $\mathrm{PCa}$, and works by targeting microtubules, altering the efflux pump in the cell (so that drug remains in the cell longer), and inducing apoptosis. ${ }^{12}$ Carboplatin (a platinum-based drug), and everolimus (an mTOR inhibitor) have been used in clinical trials as a combination therapy and have been shown to exert antitumor effects and to reduce prostate-specific antigen (PSA) levels. ${ }^{7}$

Chemotherapy agents such as docetaxel can be beneficial for many patients with metastatic CRPC; however, more specific bone-targeting agents could help eliminate the adverse effects to other non-cancerous replicating cells throughout the patient's body. In general, docetaxel is well tolerated by patients, but it can result in fever, fatigue, pneumonitis, gastrointestinal complications, neuropathies and more. ${ }^{13}$

\section{Immune therapy}

\section{Sipuleucel-T}

Sipuleucel-T has recently been approved by the U.S. FDA as an antigen presenting cell (APC)-based vaccine for PCa. ${ }^{6}$ Sipuleucel-T works through $\mathrm{T}$ cell immunity targeted against PCa cells, and utilizes peripheral blood mononuclear cells, including APCs, from the patient. These cells are activated ex vivo with a recombinant fusion protein (PA2024) consisting of a prostate antigen, prostatic acid phosphatase (PAP), fused to the granulocyte-macrophage colony-stimulating factor. This therapy acts through APC stimulation of $\mathrm{T}$ cell immune responses targeted to PAP that is highly expressed on most PCa cells. The prostate antigen is incubated with patient APCs (obtained by blood draw) and infused back into the patient once the cells are activated and ready to fight the PCa cells.

Sipuleucel-T has been shown to reduce the risk of death among metastatic CRPC patients and to increase the median survival by at least 4 months, as compared to other currently available therapeutics. ${ }^{14,15}$ Adverse effects for this immunotherapy are similar to flu-like symptoms, and include headache, fever, nausea, chills, joint pain, and some gastrointestinal discomfort. But unlike with the other treatments, these symptoms usually occur immediately following application of the drug and rescind within a couple of days. The extended survival (of at least 4 months as compared to the other drugs), the better quality of life during treatment, and the reduced risk of death by $22.5 \%$ could make this therapy worth the time and money for many patients with advanced or metastatic CRPC. ${ }^{15}$

\section{Immune checkpoint therapy}

Immune checkpoint therapy is another treatment method that has been in clinical trials for multiple cancers, including PCa. These kinds of therapies utilize various antibody targets involved in immune response to elicit antitumor effects through T cells. ${ }^{16}$ Nivolumab and pembrolizumab are antibodies against the programmed death-1 (PD-1) protein that promote cytotoxic T cell immunity to increase the attack on and destruction of cancer cells. Ipilimumab is another immune checkpoint therapy that has been in clinical trials, which targets the cytotoxic T lymphocyte-associated protein 4 (CTLA-4). This therapy works similarly by inducing adaptive immunity so that $\mathrm{T}$ cells become activated and begin attacking PCa cells. ${ }^{16,17}$

\section{Chimeric antigen receptor (CAR) T cell therapy}

CAR T cell therapy is another novel treatment used in various cancer types. This type of therapy targets cancer-specific antigens in order to gain specificity and kill cancer cells solely. The prostatespecific membrane antigen (PSMA) and prostate stem cell antigen (PSCA) have been targeted in clinical trials for $\mathrm{PCa}$, but have shown minimal antitumor effects. ${ }^{18}$

More advanced CAR T cell therapies use a combination of costimulatory agents, and these have shown much greater antitumor effects than the first-generation therapies; however, they have also shown a greater potential for eliciting more severe adverse immune reactions. Cytokine release syndrome remains a concern for CAR T cell therapy, as it can lead to fevers, fatigue, nausea, and the life-threatening conditions of multi-organ dysfunction or failure. ${ }^{19,20} \mathrm{PCa}$ expresses many antigens that are not present in other tissues, which is what makes this type of therapy so attractive, and once the adverse effects of these drugs are diminished, the development of combined immunotherapies targeting these antigens will have substantial antitumor potential for both metastatic and castration-resistant disease.

\section{Targeting the bone microenvironment}

\section{Bisphosphonates}

While chemotherapies, ADT, and radiation may help reduce progression of disease, there is still need for therapy that can treat bone metastasis specifically. There are currently several drugs available that target the bone microenvironment so that normal bone mass can be achieved.

Bisphosphonates are synthetic analogues of phosphates that inhibit hydroxyapatite crystal dissolution in bone tissues. These drugs are endocytosed by osteoclasts and become incorporated into the cell as non-hydrolyzable analogues of adenosine triphosphate (ATP), which accumulates in the cell, inhibits absorption, and induces apoptosis. Targeting osteoclasts leads to reduction in bone resorption and decreased bone turnover, which is increased in metastases. There are 3 different classes of bisphosphonates used in the clinic, and the second- and third-generations include nitrogen-containing bisphosphonates, like zoledronic acid.

Zoledronic acid was found to be 10,000 -fold more potent than first-generation bisphosphonates, such as clodronate. ${ }^{21}$ Zoledronic acid improves upon other bisphosphonates by additionally inhibiting farnesyl diphosphate synthase, which blocks the mevalatone pathway. This reduces downstream metabolites and prevents post- 
translational modifications of GTPases like Ras, Rab, Rho, and Rac that are essential signaling proteins for regulation of multiple cell processes in osteoclasts. ${ }^{21}$ Bisphosphonates bind to the bone matrix and are absorbed by osteoclasts during bone remodeling that occurs during metastasis, and the interference with their maturation leads to apoptosis. This reduces osteoclast function and inhibits resorption, leading to overall reduction in bone turnover, osteoblast function, and bone mass maintenance. ${ }^{22,23}$ While the bisphosphonates can help slow disease progression and reduce bone pain, there still remains a need for more curative treatment.

\section{RANKL inhibitors}

Denosumab has been developed as an alternative therapy to bisphosphonates, and functions by inhibiting RANK signaling. It is a monoclonal antibody to RANK ligand (RANKL), and has been shown to increase time to skeletal-related events (SREs) better than bisphosphonates. ${ }^{24}$ Blocking RANKL prevents mature osteoclast activity as well as their differentiation, which interrupts bone turnover. ${ }^{21}$ Denosumab was found to be a superior treatment to zoledronic acid for time to first SRE, with the time being increased $18 \%$ (36 months), but without any significant change in overall survival or disease progression. It did, however, decrease serum levels of urine N-telopeptide (uNTx) and alkaline phosphatase (ALP) as compared to zoledronic acid treatment. uNTx is a bone turnover marker that has been associated with increased risk in patients with PCa. In particular, patients with moderate to high levels of uNTx were shown to have a 2-fold increased risk for skeletal complications and disease progression; thus, a drug that results in lower levels of this marker is expected to significantly reduce risk for PCa advancement. ${ }^{21,25}$

There are particular benefits to the two bone-targeted therapies described above, and each patient will have a different case that may benefit from either one. Zoledronic acid needs to be intravenously administered and patients are monitored for renal complications throughout treatment. Denosumab can be given subcutaneously or orally and has no adverse effects in renal health or acute phase reactions. Patients will need to consider the cost of treatment along with long-term adverse effects and benefits depending on their disease severity and age.

\section{Targeting PCa and bone microenvironment}

\section{Radiation}

Radiation therapy after radical prostatectomy is standard of care for high-risk and metastatic PCa. ${ }^{3}$ Radiation localized to the pelvic region, as mentioned previously, is an option for patients that may be at risk for micrometastases at time of diagnosis. This can help prevent further growth in the pelvis as well as potential spread to other bones. There has been evidence to suggest that radiation not only treats $\mathrm{PCa}$ metastases, but also affects the bone microenvironment so that the bone is no longer hospitable to metastasis. Treatment with radiopharmaceuticals could potentially keep PCa cells or bone metastases dormant by inhibiting the necessary bone factors that lead to disease progression. ${ }^{5}$ This treatment can be a useful tool for CRPC by targeting mechanisms other than AR signaling, but other damaging effects will occur with treatment.

Radium-223 is an alpha emitting radiopharmaceutical used for PCa bone metastasis. It has a structure similar to calcium that helps it target the bone microenvironment and is less damaging than beta or gamma emissions. ${ }^{24,26}$ A significant improvement in overall survival of at least 4 months has been seen in CRPC bone metastasis patients. ${ }^{24}$ Multiple studies have been performed with this therapy, and have produced mixed results as to whether survival and disease progression are affected, but, overall, patients that have aggressive CRPC with a greater number of bone metastases have longer progression-free survival than those without Radium-223 treatment. ${ }^{5}$ Based on these results, it seems that this therapy may be a good choice for patients with aggressive PCa that has progressed to CRPC with multiple metastases.

\section{Conclusion and perspectives}

The continued development of new treatments for advanced PCa is promising for the future of patient therapy. Combination therapies targeted to the bone as well as new immunotherapies, such as sipuleucel-T and CAR T cell therapy have the potential to significantly reduce disease progression in $\mathrm{PCa}$ patients. Two of the biggest hurdles yet to be overcome are the adverse reactions that result from these drugs and the need to find a way by which to determine which therapy is best for each specific case.

There are currently 90 phase I/II trials and several phase III trials available for PCa patients. ${ }^{27}$ Vascular endothelial growth factor (VEGF) and fibroblast growth factor receptor (FGFR) inhibitors are a recent development designed to target angiogenesis in $\mathrm{PCa}$ tumors. A serine-arginine protein kinase 1 (SRPK1) inhibitor that switches VEGF splicing to antiangiogenic isoforms, rather than angiogenic ones, has shown improvement in PCa patient-derived xenograft models. This inhibitor has reduced tumor growth and microvessel formation in mouse tumors, and the SR proteins that the inhibitor regulates are up-regulated in human $\mathrm{PCa}$ tissues in correlation with disease stage and invasion. Drugs like alibercept, pasopanib, dovitinib, and lucitanib are currently in clinical trials, targeting VEGF and FGF pathways, but have not shown much improvement over the current therapies as of yet. ${ }^{28}$

There are many pathways and possible targets for PCa bone metastasis, and after reviewing the past and current therapies, combination treatment seems to be the most aggressive and successful treatment method for advanced and metastatic CRPC. Although combination therapy can have adverse side effects, the patients must compare the quality of life they expect during therapy to the expected survival and potential for disease progression. Each patient will present a different case, and depending on age and disease severity, clinicians will have to decide which therapy would be more beneficial for each one. With the new therapies that have been approved in the past several years and the treatments continuously entering the pipeline, there is hope for the future of metastatic CRPC therapy. But, as the current drugs only prolong the inevitable, there is still a need for a more curative treatment approach.

\section{Conflict of interest}

The authors have no conflict of interests related to this publication.

\section{Author contributions}

Conception and design (AVA, XL), writing of the manuscript (AVA, EW, XL), supervision (XL). 


\section{References}

[1] Li X, Sterling JA, Fan KH, Vessella RL, Shyr Y, Hayward SW, et al. Loss of TGF-beta responsiveness in prostate stromal cells alters chemokine levels and facilitates the development of mixed osteoblastic/osteolytic bone lesions. Mol Cancer Res 2012;10(4):494-503. doi:10.1158/1541-7786.

[2] Canalichio K, Jaber Y, Wang R. Surgery and hormonal treatment for prostate cancer and sexual function. Transl Androl Urol 2015;4(2):103-109. doi:10.3978/j.issn.2223-4683.2015.01.13.

[3] Rove KO, Crawford ED. Traditional androgen ablation approaches to advanced prostate cancer: new insights. Can J Urol 2014;21(2 Supp 1):14-21.

[4] Juloori A, Shah C, Stephans K, Vassil A, Tendulkar R. Evolving Paradigm of Radiotherapy for High-Risk Prostate Cancer: Current Consensus and Continuing Controversies. Prostate Cancer 2016;2016:2420786, doi:10.1155/2016/ 2420786.

[5] Bilen MA, Johnson MM, Mathew P, Pagliaro LC, Araujo JC, Aparicio A, et al. Randomized phase 2 study of bone-targeted therapy containing strontium- 89 in advanced castrate-sensitive prostate cancer. Cancer 2015;121(1):69-76. doi:10.1002/cncr.28971.

[6] DeSantis CE, Lin CC, Mariotto AB, Siegel RL, Stein KD, Kramer JL, et al. Cancer treatment and survivorship statistics, 2014. CA Cancer J Clin 2014;64(4):252-271. doi:10.3322/caac.21235.

[7] Yoo S, Choi SY, You D, Kim CS. New drugs in prostate cancer. Prostate Int 2016;4(2):37-42. doi:10.1016/j.prnil.2016.05.001.

[8] Antonarakis ES, Lu C, Wang H, Luber B, Nakazawa M, Roeser JC, et al. AR$\mathrm{V} 7$ and resistance to enzalutamide and abiraterone in prostate cancer. N Engl J Med 2014;371(11):1028-1038. doi:10.1056/NEJMoa1315815.

[9] Ryan CJ, Smith MR, Fizazi K, Saad F, Mulders PF, Sternberg CN, et al. Abiraterone acetate plus prednisone versus placebo plus prednisone in chemotherapy-naive men with metastatic castration-resistant prostate cancer (COU-AA-302): final overall survival analysis of a randomised, doubleblind, placebo-controlled phase 3 study. Lancet Oncol 2015;16(2):152-160. doi:10.1016/S1470-2045(14)71205-7.

[10] Liu C, Armstrong C, Zhu Y, Lou W, Gao AC. Niclosamide enhances abiraterone treatment via inhibition of androgen receptor variants in castration resistant prostate cancer. Oncotarget 2016;7(22):32210-32220. doi:10.18632/oncotarget.8493.

[11] Chandrasekar T, Yang JC, Gao AC, Evans CP. Mechanisms of resistance in castration-resistant prostate cancer (CRPC). Transl Androl Urol 2015;4(3):365380. doi:10.3978/j.issn.2223-4683.2015.05.02.

[12] Tsao CK, Cutting E, Martin J, Oh WK. The role of cabazitaxel in the treatment of metastatic castration-resistant prostate cancer. Ther Adv Urol 2014;6(3):97104. doi: $10.1177 / 1756287214528557$.

[13] Ho MY, Mackey JR. Presentation and management of docetaxel-related adverse effects in patients with breast cancer. Cancer Manag Res 2014;6:253-259. doi:10.2147/CMAR.S40601.
[14] Kantoff PW, Higano CS, Shore ND, Berger ER, Small EJ, Penson DF, et al. Sipuleucel-T immunotherapy for castration-resistant prostate cancer. N Engl J Med 2010;363(5):411-422. doi:10.1056/NEJMoa1001294.

[15] Anassi E, Ndefo UA. Sipuleucel-T (provenge) injection: the first immunotherapy agent (vaccine) for hormone-refractory prostate cancer. P T 2011;36(4):197202.

[16] Sharma P, Allison JP. Immune checkpoint targeting in cancer therapy: toward combination strategies with curative potential. Cell 2015;161(2):205-214. doi:10.1016/j.cell.2015.03.030.

[17] Carlino MS, Long GV. Ipilimumab Combined with Nivolumab: A Standard of Care for the Treatment of Advanced Melanoma?. Clin Cancer Res 2016;22(16):3992-3998, doi:10.1158/1078-0432.CCR-15-2944.

[18] Hillerdal V, Essand M. Chimeric antigen receptor-engineered T cells for the treatment of metastatic prostate cancer. BioDrugs 2015;29(2):75-89. doi:10.1007/s40259-015-0122-9.

[19] Kloss CC, Condomines M, Cartellieri M, Bachmann M, Sadelain M. Combinatorial antigen recognition with balanced signaling promotes selective tumor eradication by engineered T cells. Nat Biotechnol 2013;31(1):71-75. doi:10.1038/nbt.2459.

[20] Maude SL, Barrett D, Teachey DT, Grupp SA. Managing cytokine re lease syndrome associated with novel $\mathrm{T}$ cell-engaging therapies. Cancer $\mathrm{J}$ 2014;20(2):119-122. doi:10.1097/PPO.0000000000000035.

[21] Gampenrieder SP, Rinnerthaler G, Greil R. Bone-targeted therapy in metastatic breast cancer - all well-established knowledge?. Breast Care (Basel) 2014;9(5):323-330. doi:10.1159/000368710.

[22] El-Amm J, Aragon-Ching JB. Targeting Bone Metastases in Metastatic Castration-Resistant Prostate Cancer. Clin Med Insights Oncol 2016(Suppl 1):11-19. doi:10.4137/CMO.S30751.

[23] Ottanelli S. Prevention and treatment of bone fragility in cancer patient. Clin Cases Miner Bone Metab 2015;12(2):116-129. doi:10.11138/ccmbm/2015.12. 2.116 .

[24] Cook LM, Shay G, Araujo A, Lynch CC. Integrating new discoveries into the "vicious cycle" paradigm of prostate to bone metastases. Cancer Metastasis Rev 2014;33(2-3):511-525. doi:10.1007/s10555-014-9494-4.

[25] Fizazi K, Carducci M, Smith M, Damiao R, Brown J, Karsh L, et al. Denosumab versus zoledronic acid for treatment of bone metastases in men with castration-resistant prostate cancer: a randomised, double-blind study. Lancet 2011:377(9768):813-822 doi:10.1016/S0140-6736(10)62344-6.

[26] Sridhar SS, Joshua AM, Gregg R, Booth CM, Murray N, Golubovic J, et al. A phase II study of GW786034 (pazopanib) with or without bicalutamide in patients with castration-resistant prostate cancer. Clin Genitourin Cancer 2015;13(2):124-129. doi:10.1016/j.clgc.2014.06.001.

[27] Prostate Cancer Foundation. Available from: www.pcf.org. Accessed August 2016.

[28] Mavrou A, Oltean S. SRPK1 inhibition in prostate cancer: A novel anti-angiogenic treatment through modulation of VEGF alternative splicing. Pharmacol Res 2016;107:276-281. doi:10.1016/j.phrs.2016.03.013. 